\title{
Publisher Correction: Learning MRI artefact removal with unpaired data
}

Siyuan Liu(D), Kim-Han Thung (10), Liangqiong Qu, Weili Lin, Dinggang Shen and Pew-Thian Yap (i)

Correction to: Nature Machine Intelligence https://doi.org/10.1038/s42256-020-00270-2, published online 19 January 2021.

In the version of this Article originally published, the sentence 'The authors would also like to thank D. Shen for an initial discussion of the problem when he was with the University of North Carolina at Chapel Hill' at the end of the Acknowledgements section was included by mistake; it should have been removed. Also, the text 'D.S. was involved in the initial discussion of the problem when he was with the University of North Carolina at Chapel Hill' was missing from the Author contributions section; it should have been included before the last sentence. This has now been corrected.

Published online: 27 January 2021

https://doi.org/10.1038/s42256-021-00300-7

(C) The Author(s), under exclusive licence to Springer Nature Limited 2021 